Journal Club

Editor's Note: These short, critical reviews of recent papers in the Journal, written exclusively by graduate students or postdoctoral fellows, are intended to summarize the important findings of the paper and provide additional insight and commentary. For more information on the format and purpose of the Journal Club, please see http://www.jneurosci.org/misc/ifa_features.shtml.

\title{
Tonotopic Organization of V5/MT + in Congenital Anopthalmia: Implications for Auditory Motion Processing and Metamodal Cross-Modal Reorganization
}

\author{
Martha M. Shiell \\ Integrated Program in Neuroscience, McGill University, Montreal, Quebec H3A 2B4, Canada \\ Review of Watkins et al.
}

The study of cross-modal reorganization after sensory deprivation can be a powerful strategy for understanding the functional structure of the brain. This strategy rests on the hypothesis that the brain follows a metamodal organization, with specific regions or networks responsible for specific computational functions, regardless of the modality of the sensory input (Pascual-Leone and Hamilton, 2001). A simple example of this concept is illustrated in functional neuroimaging studies of area $\mathrm{V} 5 / \mathrm{MT}+$, the well established visual motion processing region. Consistent with its role in vision, $\mathrm{V} 5 / \mathrm{MT}+$ responds to auditory motion after blindness (for review, see Voss and Zatorre, 2012). This cross-modal motion sensitivity has been interpreted as preserved functional output after visual deprivation, and is thus consistent with the hypothesized function of $\mathrm{V} 5 / \mathrm{MT}+$ in motion processing.

A recent study on auditory processing after blindness introduces a potential complication to the straightforward interpretation of cross-modal reorganization in V5/MT+ (Watkins et al., 2013). Using functional magnetic resonance imaging,

Received Jan. 13, 2014; revised Feb. 6, 2014; accepted Feb. 12, 2014.

I thank R. Zatorre and P. Voss for comments on a previous draft of this manuscript.

Correspondence should be addressed to Martha M. Shiell, Montreal Neurological Institute, 3661 University Street, Montreal QC H3A 2B4. E-mail: martha.shiell@mail.mcgill.ca.

DOI:10.1523/JNEUROSCI.0150-14.2014

Copyright $\odot 2014$ the authors $\quad 0270-6474 / 14 / 343807-03 \$ 15.00 / 0$
Watkins et al. (2013) compared auditory processing in six sighted and five blind adults with congenital anopthalmia, a condition in which the eye fails to develop. Participants listened to pure-tone auditory trains in the high, middle, and low frequencies. The blood oxygen leveldependent (BOLD) signal during these conditions was contrasted with that during silence. Compared with the sighted control group, the anopthalmic group showed increased activity in the posterior temporal/lateral occipital cortex, a region that overlapped entirely with a cytoarchitectonically defined probabilistic map of $\mathrm{V} 5 / \mathrm{MT}+$. The researchers further examined the organization of this auditory response by assigning each active voxel to the frequency condition that elicited the greatest proportion of that voxel's activity. In the three anopthalmic participants who showed the greatest auditory response in $\mathrm{V} 5 / \mathrm{MT}+$, the activation followed a tonotopic organization. This organization supports the suggestion that the observed activation reflects early auditory processing of frequency information, rather than an unspecific coactivation.

The frequency sensitivity of V5/MT+ in anopthalmic people is somewhat surprising considering the well established involvement of this region in auditory motion processing after blindness (Voss and Zatorre, 2012). As spectral cues are relevant for auditory spatial processing, frequency and auditory motion process- ing may be compatible, but it is not clear if or how the two processes relate. Since the auditory motion response of previous research was identified within a primarily non-anopthalmic blind population, whether or not it generalizes to the anopthalmic group remains to be determined. Anopthalmia, unlike most other etiologies of blindness, ensures no activity in the visual system from either light or endogenous retinal firing, and may therefore result in a different pattern of reorganization than that which occurs through non-anopthalmic blindness. While it is possible that frequency and motion processing are both localized to V5/MT+ in anopthalmics, it is also possible that V5/ $\mathrm{MT}+$ of this group has deviated from its motion processing role. These two alternatives each have important implications for our understanding of brain function: the former for auditory motion processing, and the latter for the metamodality of the brain.

The neural mechanisms of auditory motion processing are not well understood. Numerous studies have identified a network of human cortical regions involved in this process, including the planum temporale (Baumgart et al., 1999), the right hemisphere posterior parietal cortex, and the bilateral prefrontal cortices (Griffiths et al., 1998). Of these structures, tonotopy is found solely in subregions of the planum temporale (Humphries et al., 2010), but the 
relevance of this organization for motion processing remains unexplored. As such, we can only speculate on plausible roles for tonotopy in the putative motion processing of $\mathrm{V} 5 / \mathrm{MT}+$. One possibility is that tonotopy facilitates the processing of spectral features of auditory motion. Spectral spatial cues arise from small distortions in sound that are introduced by the listener's body, particularly the pinna of the ear. Previous research shows that blind people rely on spectral cues for their superior spatial localization skills, and that these cues are processed cross-modally (Voss et al., 2011). Likewise, V5/MT+ may have reorganized to process spectral cues for auditory motion. If supported, this form of reorganization would illustrate a previously unknown overlap between auditory frequency and motion processing, and thus help elucidate the cortical mechanism of the latter. Given that spectral cues are particularly relevant for vertical sound localization, one prediction from this hypothesis is that anopthalmic people will show enhanced detection or discrimination of spatially ascending and descending sounds, and that this ability will correlate with crossmodal activity in V5/MT+.

Despite the unknowns surrounding auditory motion processing, it is clear that motion in the auditory domain is different from its counterpart in vision. Notably, there is no evidence for an auditory homolog to the visual area V5/MT+ (Smith et al., 2007). This difference between the auditory and visual modalities is not surprising when we consider how motion is encoded in the two sensory systems: while visual motion is encoded from spatial changes over the retina, auditory motion is encoded from spectral information and binaural differences in timing and amplitude. Some have argued that the parallel to visual motion is not auditory motion, but rather spectral motion, that is, changes in frequency over time (Thivard et al., 2000). This idea arises from the observation that both visual and spectral motion are encoded by the movement of energy across a field of sensory receptors. Although the importance of tonotopy for spectral motion processing remains unconfirmed, we can predict its relevancy with the analogy between the two types of motion: whereas visual motion processing regions are organized topographically according to the retina, spectral motion processing regions should organize topographically according to the basilar membrane. Following the hypothesis of a metamodal organization of the brain, the correspondences between spectral and visual motion might lead to the expectation that V5/MT+ will reorganize to process auditory spectral motion, rather than spatial motion, after visual deprivation. This possibility could be explored further by comparing the neural activity in V5/MT+ that is elicited by these two different types of auditory stimuli in anopthalmic people.

While the substitution of auditory motion, be it spatial or spectral, for visual motion is a possible explanation for the V5/MT+ activation found by Watkins et al. (2013), we require a better understanding of the role of tonotopy in these functions before evaluating their plausibility. An alternative hypothesis is that the results of Watkins et al. (2013) reflect reorganization that is unrelated to motion processing. This scenario challenges the overarching theory of cross-modal reorganization after sensory deprivation: that function is preserved (Pascual-Leone and Hamilton, 2001). A similar challenge to this theory has been raised by the results of another recent study of auditory motion processing in blind people. Using electroencephalography, Lewald and Getzmann (2013) uncovered an extensive network of visual regions that responded to auditory motion in blind people more than in sighted, illustrating that cross-modal motion processing was not limited to V5/ $\mathrm{MT}+$. This finding was interpreted as evidence that auditory and visual motion processing are not interchangeable functions. The experimenters suggested that, since hearing participants also demonstrate auditory motion sensitivity in V5/ $\mathrm{MT}+$ (Poirier et al., 2005), the crossmodal activity of this region in blind people might represent an expansion of an already present function that is complementary but not analogous to visual motion processing (Lewald and Getzmann, 2013). For example, in normalsighted people, visually sensitive neurons in V5/MT + might be modulated by auditory input. After blindness, this modulatory input could expand, but not to the point where it replaced the missing visual input in the primary mechanisms of V5/ $\mathrm{MT}+$. This interpretation is subtly different from that offered by the metamodal perspective, which implies that the auditory and visual inputs into V5/MT+ are mechanistically interchangeable.

In the Lewald and Getzmann (2013) study, the largest difference in auditory motion-related activity between the blind and sighted groups occurred in the ante- rior calcarine sulcus, a region that represents the peripheral visual field in the primary visual cortex of sighted individuals. Interestingly, the same region showed a subthreshold activation in the anopthalmic group of Watkins et al. (2013). In one anopthalmic case, this activation also followed a tonotopic organization. Although this finding requires replication and both effects need to be demonstrated across the different blind populations, the trend suggests that auditory frequency and spatial motion processing reorganize in parallel after visual deprivation, again alluding to an auditory motion mechanism that overlaps with frequency processing.

The findings of Watkins et al. (2013) illustrate the potential of sensory deprivation as a tool for understanding the functional structure of the brain. Following the assumption of a metamodal organization, these findings have implications for our understanding of the relationship between frequency and motion processing in the auditory system, and call attention to the need for more research on the neural mechanisms for processing spectral spatial cues and spectral motion. Together with recent research on auditory motion processing in blind people (Lewald and Getzmann, 2013), these findings may also lead to a re-evaluation of currently held beliefs about the metamodal structure of the brain, particularly with regards to the putative correspondence between auditory and visual motion processing. Regardless of what explanation for these findings prevails, the further study of cross-modal activity in congenital anopthalmia promises exciting consequences for theories of the function and organization of the brain.

\section{References}

Baumgart F, Gaschler-Markefski B, Woldorff MG, Heinze HJ, Scheich H (1999) A movement-sensitive area in auditory cortex. Nature 400:724-726. CrossRef Medline

Griffiths TD, Rees G, Rees A, Green GG, Witton C, Rowe D, Büchel C, Turner R, Frackowiak RS (1998) Right parietal cortex is involved in the perception of sound movement in humans. Nat Neurosci 1:74-79. CrossRef Medline

Humphries C, Liebenthal E, Binder JR (2010) Tonotopic organization of human auditory cortex. Neuroimage 50:1202-1211. CrossRef Medline

Lewald J, Getzmann S (2013) Ventral and dorsal visual pathways support auditory motion processing in the blind: evidence from electrical neuroimaging. Eur J Neurosci 38:3201-3209. CrossRef Medline

Pascual-Leone A, Hamilton R (2001) The metamodal organization of the brain. In: Vision: from neurons to cognition (Progress in brain 
research, vol. 134) (Casanova C, Ptito M, eds), pp 427-444. Philadelphia: Elsevier.

Poirier C, Collignon O, DeVolder AG, Renier L, Vanlierde A, Tranduy D, Scheiber C (2005) Specific activation of the V5 brain area by auditory motion processing: an fMRI study. Brain Res Cogn Brain Res 25:650-658. CrossRef Medline

Smith KR, Saberi K, Hickok G (2007) An eventrelated fMRI study of auditory motion per- ception: no evidence for a specialized cortical system. Brain Res 1150:94-99. CrossRef Medline

Thivard L, Belin P, Zilbovicius M, Poline JB, Samson Y (2000) A cortical region sensitive to auditory spectral motion. Neuroreport 11: 2969-2972. CrossRef Medline

Voss P, Zatorre RJ (2012) Organization and reorganization of sensory-deprived cortex. Curr Biol 22:R168-R173. CrossRef Medline
Voss P, Lepore F, Gougoux F, Zatorre RJ (2011) Relevance of spectral cues for auditory spatial processing in the occipital cortex of the blind. Front Psychol 2:48. CrossRef Medline

Watkins KE, Shakespeare TJ, O'Donoghue MC, Alexander I, Ragge N, Cowey A, Bridge H (2013) Early auditory processing in area $\mathrm{V} 5 / \mathrm{MT}+$ of the congenitally blind brain. J Neurosci 33:18242-18246. CrossRef Medline 